\title{
Dynamic Social Simulation with Multi-Agents having Internal Dynamics
}

\author{
Takashi Sato* and Takashi Hashimoto \\ School of Knowledge Science, \\ Japan Advanced Institute of Science and Technology (JAIST), \\ Tatsunokuchi, Ishikawa, Japan, 923-1292 \\ \{stakashi, hash\}@jaist.ac.jp \\ $+81-761-51-1882$
}

Submitted to: Artificial Life

\begin{abstract}
In this paper, we discuss a viewpoint to regard individuals in a society as agents having internal dynamics, in order to study the dynamical nature of social structures. Internal dynamics is the autonomous changes of internal states upon which an agent's behavior depends. By introducing the notion of internal dynamics, we propose a simple recurrent network with self-influential connection (SRN-SIC) as a model of an agent for dynamic social simulation. We report the simulation results of a multi-agent system, in which the agents play the minority game as a social interaction. The system has a micro-macro loop, namely, individual behaviors (micro) interact with social states (macro). In the simulation, we observed the dynamics of a macro structure itinerated among ordered states via disordered states. Several analyses support the importance of internal dynamics and micro-macro loop for forming and maintaining dynamic macro structure. We conclude that the internal dynamics of agents and micro-macro loop should be introduced to construct dynamic social simulations.
\end{abstract}

Keywords: Internal Dynamics, Micro-Macro Loop, Simple Recurrent Network with Self-Influential Connection, Dynamic Social Simulation, Multi-Agent System, Minority Game

${ }^{*}$ corresponding author 


\section{Introduction}

The spontaneous social structures in a society, such as institutions, classes, and markets, usually cannot be separated from individuals in the society, since individuals both shape and are influenced by the structures. The key notion when considering spontaneous structures is the micro-macro loop $[16,17]$. However, we think that this notion alone cannot explain some of the changes in social structure which are seen in an actual society. In this paper, we introduce internal dynamics in addition to the micro-macro loop and illustrate with a multi-agent simulation that both social structure at a macro level and individuals' behavior at a micro level continue to change.

In traditional economics, individuals are often assumed to be isolated from others, with independent utilities and preferences. On the other hand, Egashira and Hashimoto[3] propose the notion of socially developmental individuals whose cognitive frameworks, including utilities and preferences, are shaped through interaction among themselves. They show the emergence of an institution as a pattern of cognitive frameworks common to the individuals[8]. However, once organized, the institution in their model never changes. In general, if influences from the macro structure to the micro level have a self-enforcement function to regulate the behavior of individuals, it is thought that an institution can emerge and be maintained[1]. But, spontaneous changes of the social structure are not shown in such a case. In reality, social structure changes dynamically. Changes in the macro structure are often thought to be caused by changes coming from outside the micro-macro loop, but a mechanism of endogenous change is not explained.

In addition to the idea of socially developmental individuals, we introduce the notion of internal dynamics, representing the basic nature of cognitive individuals in a society, in order to understand the endogenous change of social structures. Internal dynamics refers to autonomous changes of the internal states of individuals. It has received attention in the field of cognitive science. An example of a cognitive phenomenon which supports the effect of internal dynamics on cognition is an experiment using reversible figures such as Necker cube. In this experiment, although the figure never changes objectively, the subjective vision of the figure changes with time. This result suggests that cognitive processing is evoked by autonomous changes in the internal state. Moreover, there are some studies about a perception of ambiguous patterns by using chaotic neural network, namely, a network with internal dynamics[13, 12].

Recent cognitive science has developed into clarifying dynamical nature of cognitive systems. Gelder, for example, advocates that humans should be regarded as a kind of dynamical systems, since the complex behavior of dynamical systems can well express cognitive phenomena[15, 7]. Varela manifests the importance of structural coupling, which appropriately connects the internal states of a cognitive system to its environment through the interaction between them[20]. Karmiloff-Smith insists that self-modification process to transform an implicit information in mind into an explicit knowledge plays an important role in human cognitive development[11]. These studies place importance on the dynamic change of cognitive systems.

A recurrent neural network which is regarded as a kind of dynamical systems agrees with Gelder's way of thinking, which treats humans as dynamical cognitive systems. The recurrent network is known to have various functions 
such as pattern recognition, motion control, and time series prediction. Examples of such neural networks are Dynamical Recognizer proposed by Pollack[14], Simple Recurrent Network (SRN) designed by Elman[4] and so on. Dynamical recognizers are used by Taiji and Ikegami as a model of an agent which forms an internal model of an opponent in iterated prisoner's dilemma game[18, 9]. In addition, Tani et al. indicate that robots having recurrent neural network show various interesting behavior such as a room navigation with 'concepts' corresponding to turning right and left at corners, which is self-organized in the recurrent network[19]. It is often used in the field of computational cognitive science. Although recurrent networks are useful in realizing various behaviors, it is difficult to treat the large number of agents necessary for social simulations, since the computational cost of the usual recurrent network is large. On the other hand, the computational cost of the SRN is comparatively less. Furthermore, the SRN is as effective in simulating dynamic cognitive and developmental psychological phenomena, since it has a powerful ability to predict a time series, and can concretely illustrate human behavior such as language comprehension $[4,5,6]$. In spite of these advantages, the SRN is not often used in social simulations. In this study, we propose a model of an agent for dynamic social simulation using SRN with modification. Here, a simulation showing the dynamics of social structure is referred to as a dynamic social simulation.

The purpose of this study is to illustrate the importance of viewing individuals in a society as cognitive agents having internal dynamics. In this paper, we perform the followings. 1) We propose a simple model of an agent having internal dynamics. The agent model is expressed as a modification of the SRN, since the internal dynamics is thought to be vital for the dynamics of social structure. In this study, we discuss how internal dynamics is important in considerations of human behavior. 2) We construct a dynamic social simulation by a multi-agent system which is composed of the agents proposed in 1). This system features the micro-macro loop, since it is conceivable that the loop plays a significant role in the dynamics of social structure. We adopt the minority game as a social interaction among the agents. 3) By means of this dynamic social simulation, we study what causes dynamics of social structure, especially how the internal dynamics in the agents and the micro-macro loop in the social system work.

The rest of the paper is organized as follows. In section 2 , we discuss the internal dynamics and propose a model of agent having internal dynamics. In section 3, a multi-agent system for dynamic social simulation is introduced. Results of the simulations are depicted in section 4 . We present a discussion of the results in section 5 and deliver our conclusions in section 6 .

\section{$2 \quad$ Internal Dynamics}

\subsection{Importance of Internal Dynamics}

From a mechanistic viewpoint, humans can be regarded as a kind of state transition machine. They have internal states that change with external stimuli and return some responses that have one-to-one correspondences with the stimuli.

However, this viewpoint is not always appropriate, since the internal state of humans does not change only in response to external stimuli. It is difficult 
to explain such characteristics of human behavior as diversity and consistency by regarding humans as mere state transition machines. The term diversity means here that humans can and often do show various behaviors in the same situation. The sequence of behaviors is usually not random, but has a certain causality. We call this feature of human behavior consistency.

The internal states of humans change even in situations in which the same external stimulus is constantly given or when no external stimulus is given. We refer to this autonomous change of the internal state as internal dynamics. By taking the internal dynamics into consideration, we can account for many features of human behavior. Humans can behave variously, even if the same stimulus is given, since their internal state, on which their behavior depends, changes autonomously. Accordingly, the human can form a one-to-many relationship among a stimulus and his/her responses by means of internal dynamics. In fact, diversity of actions is realized based on the one-to-many relationship formed internally. The internal state changes with action as well as the external situation. That is, various influences from past actions, internal states, and external stimuli are stored in the current internal state. Thus, consistency of the human behavior arises, since the actions which are based on the internal state correlate with a history of past internal states.

\subsection{Formalization of Internal Dynamics}

To propose a model of an agent having the internal dynamics, we formulate the internal dynamics in a mathematical form.

Basically, we can suppose that an agent's action, which is a time dependent variable, depends on his/her internal state. This dependency is written as

$$
a(t)=F(x(t)),
$$

where $a(t)$ is the agent's action at time $t, x(t)$ is an internal state of the agent at time $t$, and $F$ is a function to decide how the agent behaves when he/she has certain internal state. As we discussed above, the internal state changes autonomously. Thus, the dynamics of the internal state is expressed as

$$
x(t)=G(x(t-1)),
$$

by using a function $G$. In addition, the internal state is affected by the agent's past action and the present external stimulus. Therefore, the equation (2) is rewritten as

$$
x(t)=G(x(t-1), a(t-1), s(t)),
$$

where $s(t)$ is an external stimulus that the agent receives at time $t$. The equation (3) means that the internal state stores a history of past internal states, actions and external stimuli, and the equation (1) means that the history determines the present action. A schematic view showing the equations (1) and (3) is illustrated in Fig. 1. In the next section, we propose an architecture corresponding to these equations.

\subsection{Architecture of Agent Having Internal Dynamics}

In this section, we first propose a concrete agent architecture corresponding to the basic model illustrated in Fig. 1. The archtecture is a modification 


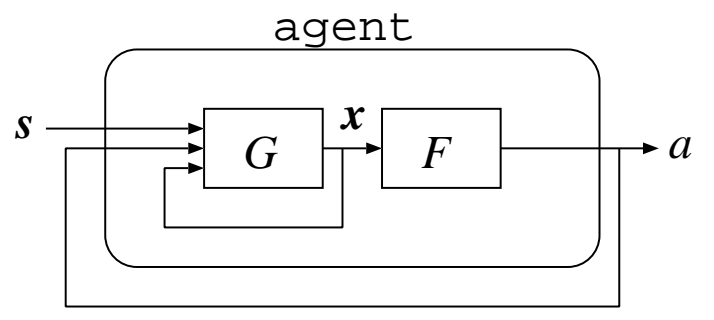

Figure 1: Schematic view of an agent having internal dynamics. The symbols $s$, $x$ and $a$ are an external stimulus, the agent's internal state and his/her action, respectively. The boxes labeled by $G$ and $F$ are functions to change the internal state and to decide how the agent behaves when he/she has a certain internal state, respectively. The arrows indicate the direction of interactions between the elements.

of the simple recurrent network (SRN) proposed by Elman[4]. We call this architecture a $S R N$ with self-influential connection $(S R N-S I C)$. Figure 2 shows the proposed architecture of the agent. The SRN has an input layer to accept external stimuli; an output layer to decide the output value based on received signals; and a hidden layer to process input values and to pass them to the output layer. Further, the SRN has a context layer in which each neuron has one-to-one connection with each neuron of the hidden layer, in order to copy a previous state of the hidden layer. Therefore, the state of the network at a certain time is decided by mixing current stimulus and a history of past states. Moreover, the SRN-SIC has an additional recurrent connection between the output and the input layer so that the agent decides his/her action based on his/her past action.

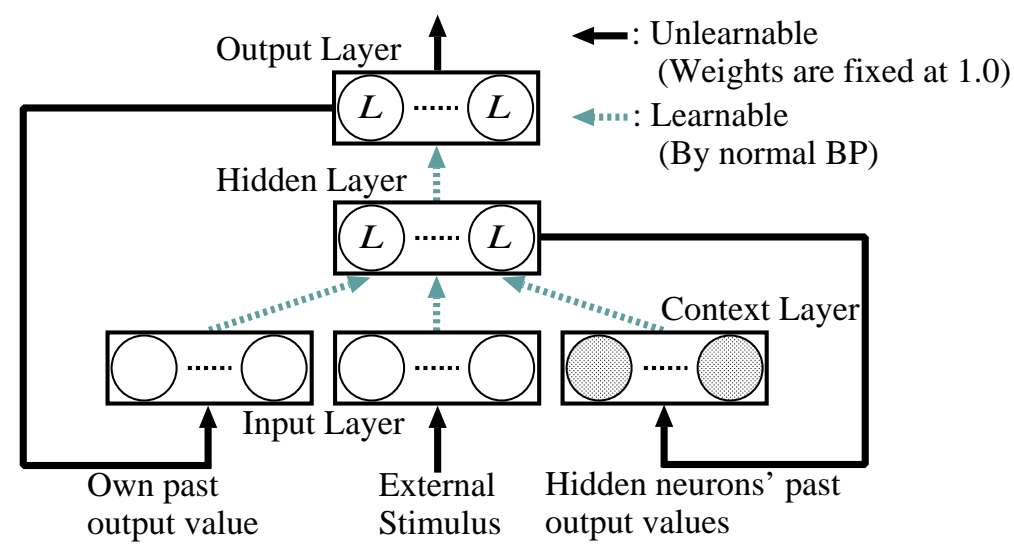

Figure 2: The SRN-SIC as the proposed architecture of the agent. This is a particular Elman-type network with an additional recurrent connection between the output and the input layer. The symbol $L$ represents a nonlinear function to output a real number between -1.0 and 1.0. Not all connections are shown. 
We show a mathematical form of the SRN-SIC. Each layer has its own index variable: $l$ for recursive output nodes, $k$ for output nodes, $j$ for hidden nodes, $i$ for input nodes, and $h$ for context nodes. The output function of each neuron other than the input and the context neurons is the differentiable nonlinear function $L$ whose range is between -1.0 and 1.0. The function $L$ is defined by

$$
L(n e t)=\tanh (\beta n e t),
$$

where net is the sum of weighted input values, and $\beta$ decides the nonlinearity of the function $L$. The output of SRN-SIC is determined by

$$
\begin{gathered}
o_{k}(t)=L\left(\operatorname{net}_{k}(t)\right), \\
\operatorname{net}_{k}(t)=\sum_{j=0} w_{k j} v_{j}(t)+\theta_{k},
\end{gathered}
$$

where $o_{k}(t)$ is the $k$-th output neuron's value at time $t, w_{k j}$ is the connection weight between the $k$-th output and the $j$-th hidden neurons, $v_{j}(t)$ is the $j$-th hidden neuron's value at time $t$, and $\theta_{k}$ is a bias of the $k$-th output neuron. The hidden neuron's activation is calculated by

$$
\begin{gathered}
v_{j}(t)=L\left(\operatorname{net}_{j}(t)\right) \\
\operatorname{net}_{j}(t)=\sum_{i=0} w_{j i} x_{i}(t)+\sum_{h=0} w_{j h} u_{h}(t)+\sum_{l=0} w_{j l} z_{l}(t)+\theta_{j}
\end{gathered}
$$

where $w_{j i}$ is the connection weight between the $j$-th hidden and the $i$-th input neurons, $x_{i}(t)$ is the $i$-th input neuron's value at time $t, w_{j h}$ is the connection weight between the $j$-th hidden and the $h$-th context neurons, $u_{h}(t)$ is the $h$-th context neuron's value at time $t, w_{j l}$ is the connection weight between the $j$-th hidden and the $l$-th recursive output neurons, $z_{l}(t)$ is the $l$-th recursive output neuron's value at time $t$, and the $\theta_{j}$ is a bias of the $j$-th hidden neuron. Each value of $u$ and $z$ can be replaced by the past hidden and the past output neuron's activation, respectively. Therefore, the equation (8) is rewritten as

$$
\operatorname{net}_{j}(t)=\sum_{i=0} w_{j i} x_{i}(t)+\sum_{h=0} w_{j h} v_{h}(t-1)+\sum_{l=0} w_{j l} o_{l}(t-1)+\theta_{j} .
$$

When we consider the context layer as a type of input layer at each time step, the network can be regarded as a kind of feedforward type neural network. Therefore, as a learning method, we adopt the error Backpropagation learning (BP). Each weight of all recurrent connections is fixed at 1.0 and is not adjusted by learning.

\section{Multi-Agent System for Dynamic Social Sim- ulation}

\subsection{Minority Game as Social Interaction}

We show a dynamic social simulation by using multi-agents with the internal dynamics proposed in the previous section. In this simulation, we adopt the 
minority game (MG), proposed by Challet and Zhang[2], as a social interaction among the agents.

The MG is characterized by the following two basic rules:

1. $N$ (odd) players must choose one out of two alternatives (-1/1 meaning buy/sell, or etc) independently at each step.

2. Those who are in the minority side win.

The MG is regarded as a model of market[21]. Namely, two alternative choices are 'sell' and 'buy.' Suppose that people want to sell or buy a product. If the number of sellers is smaller than buyers, the price of the product is higher than the opposite case. Therefore, the sellers win. Though it is very abstract model, this game can be applied to many situations in which plural but limited number of resources are utilized or shared by many people. For example, choices of two food sources or choices of two females by some males in mating.

The player in our dynamic social simulation is implemented with the SRNSIC. It has only one output neuron $o(t)$, since MG requires a move -1 or 1 . In order for the output value from the neural network to be suited for the minority game, a step function $C$ which classifies an output value into two value -1 or 1 is appended on the output neuron. The action of an agent $a(t)$ at time $t$ is given by

$$
a(t)=C(o(t))=\left\{\begin{array}{cc}
-1 & o(t)<0.0 \\
1 & o(t) \geq 0.0
\end{array} .\right.
$$

To equip our system with a micro-macro loop, we establish influence from the macro level to the micro level by the following two ways, as illustrated in Fig. 3. One is that the previous minority side is given to all players as an external stimulus at each step. The other is that all players learn a time series of the past minority side as the teacher's signal.

\subsection{Algorithm for Simulation}

The procedure of the simulation is as follows:

1. Except for initial time step, each agent independently decides a move $(-1$ or 1) based on its own past action and the minority side at the last play.

2. A current minority side is determined from all players' moves. Then, players who end up in the minority side get one point. Losers cannot gain a point.

3. The step is given an increment and go to 1 .

The procedure from 1 to 3 is called one step. By normal BP learning algorithm, all agents learn a time series of the minority side every 10,000 steps. A teacher's signal for the learning is the sequence of the minority side at the last 100 steps immediately before the learning. We refer to the 10,000 steps between the learning processes as one turn. 


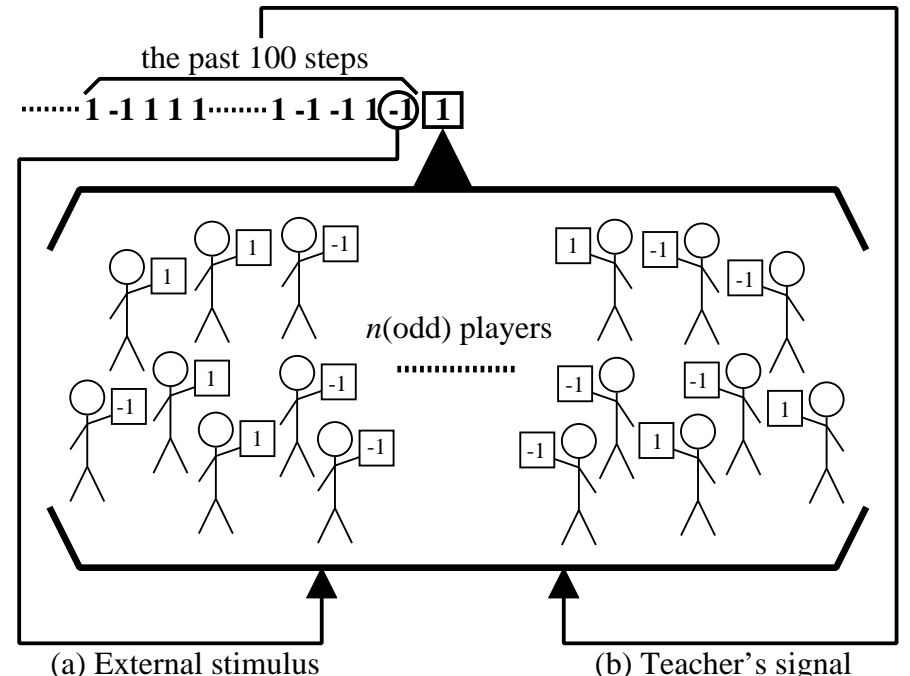

Figure 3: Propagation of influences from a macro to a micro level. (a) The past minority side is given to the agents as an external stimulus. (b) All agents learn a time series of minority side for past 100 steps.

\section{Simulation Results}

In this section, we report the results of multi-agent simulations. We distinguish here several observable dynamics in the micro and the macro levels, as indicated in Table 1.

Table 1: The range of the macro and the micro level.

\begin{tabular}{|l|l||l|l|}
\hline \multicolumn{2}{|c||}{ Macro Level } & \multicolumn{2}{c|}{ Micro Level } \\
\hline $\begin{array}{l}\text { A time series of } \\
\text { minority side }\end{array}$ & $\begin{array}{l}\text { The number of agents } \\
\text { who belong to the } \\
\text { minority side }\end{array}$ & Moves of each agent & $\begin{array}{l}\text { Internal dynamics of } \\
\text { each agent }\end{array}$ \\
\hline
\end{tabular}

Before describing the results, we list the specification of simulations. The minority game is lasted until 1,000,000 steps. The population size of the agents is 101. The SRN-SIC of each agent has one output neuron, five hidden neurons (i.e., there also are context neurons) and two input neurons. At the beginning of a simulation, all the input values including the feedback input values from the output and the hidden neurons are set to be 0.0 . The initial connection weights are set to be random real numbers between -0.5 and 0.5 , but only recurrent connection weights are fixed at 1.0. A learning process is finished when an error between teacher's signal and output of an agent became less than 0.01. As learning parameters, we set the learning rate as 0.01 , and the momentum coefficient as 0.8 . The nonlinearity strength of each output function is set to be 0.8 . 


\subsection{Dynamics at Macro Level}

\subsubsection{Classification of Change Patterns}

The time series of the minority side shows various patterns. We classify these into six, as illustrated in Fig. 4. In order to understand the dynamics at the macro level in detail, we observe in this figure the transition of minority side $(-1$ or 1) multiplied by the number of agents belonging to the minority side, namely, the winners.

(a)

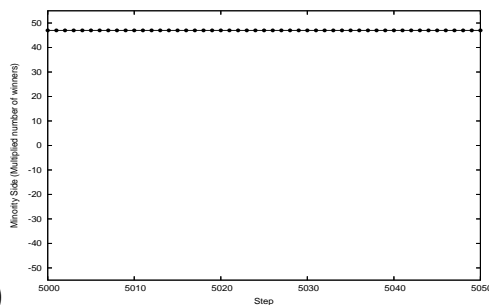

(c)

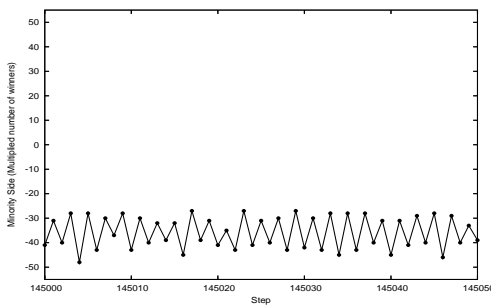

(e)

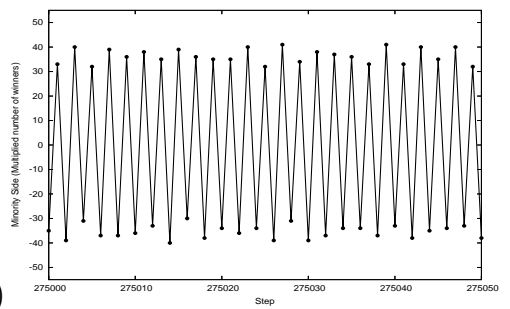

(b)

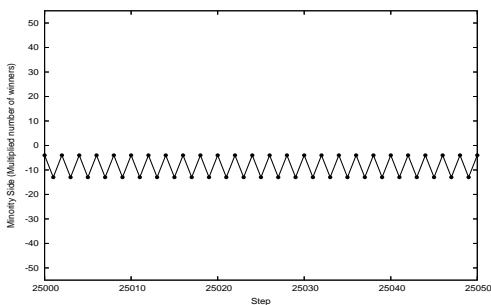

(d)

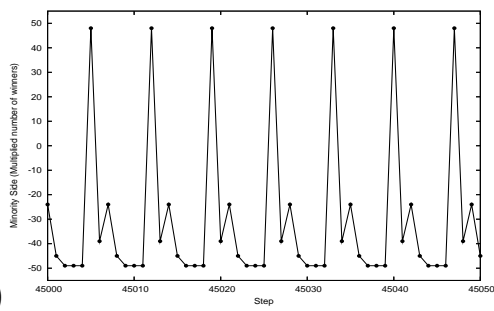

(f)

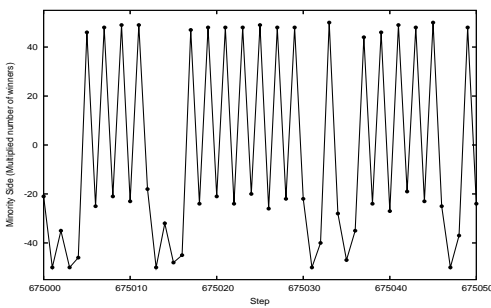

Figure 4: The patterns of time series of the minority side in different turns. The $x$-axis is the steps. The $y$-axis is the minority side (-1 or 1$)$ times the number of winners, i.e., agents in the minority side. The positive (negative) value in the $y$-axis signifies that the minority side is $1(-1)$. (a) Both the minority side and the number of winners are fixed. (b) The minority side is fixed, and the number of winners periodically changes. (c) The minority side is fixed, and the change of the number of winners is aperiodic. (d) Both the minority side and the number of winners show periodic changes. (e) The change of the minority side is periodic, and that of the number of winners is aperiodic. (f) Both the minority side and the number of winners aperiodically change.

We focus our attention on Fig. 4(b) and (c), in which the minority side never changes. Although all agents continuously receive the same external stimulus, the number of winners changes periodically in Fig. 4(b) and aperiodically in Fig. 4(c). These dynamics imply that the agents can autonomously alter the way they interpret the external information utilizing their internal dynamics, 
even if the same information is successively given to them. This resembles a human vision of a reversible figure introduced in section 1 . Note that the periodic/aperiodic changes take place in one turn in which no learning was executed.

\subsubsection{Emergence of Itinerant Dynamics}

We observe various patterns in the dynamics of the minority side, even in one turn. Figure 5 depicts a typical itinerant dynamics at the macro level in one turn $^{1}$. As can be seen, the patterns of time series of the game itinerates among various dynamical states. The transitions among fixed points and periodic changes are mediated by aperiodic dynamics ${ }^{2}$.

The dynamics illustrated in Fig. 5 is observed in one turn. That is, it is confirmed that very complex changes at the macro level are induced by the internal dynamics of each agent, though the internal structures of the agents are not modified by learning.

\subsection{Dynamics at Micro Level}

\subsubsection{Emergence of Agent's Strategy}

We showd various changes at the macro level in the previous section. The observed results suggest that the complex dynamics is formed from the agents' fruitful behavior taken place according to not only an external environment but also their internal dynamics and interaction among them. In this section, we investigate the behavior of the agents. At first, we show that agents obtain particular strategies through learning and interaction in the minority game. A strategy is a way to determine how an agent reacts to external stimuli.

Figure 6 shows two strategies, expressed as the relationship between the output value and the internal dynamics, namely, the change of two hidden neurons' values. The agent exemplified in Fig. 6(a) acquires a simple strategy which can be denoted by a deterministic finite automaton with two states. He/She behaves regularly, depending on the input values. That is to say, there is a one-to-one correspondence between the external stimulus and the action. The other type of strategy, illustrated in Fig. 6(b), accurately uses two rules depending on two kinds of input value, -1 and 1 . While the rules described by two limit cycles seem simple at a glance, the agent's behavior is complex. The points on the limit cycles are so dense that the output sequence of the agent is quasi-periodic. Further, since each limit cycle ranges over almost the entire area of the output, the strategy creates a one-to-many relationship from an input to the agent's moves. We also found agents whose strategies are expressed by finite automata with many states and complex forms like strange attractors.

\footnotetext{
${ }^{1}$ To draw the graphs in Fig. 5, we encode the time series of the minority side. At first, the minority sides, -1 and 1 , are corresponded to 0 and 1 as binary digit, respectively. Next, a 20 steps series of the minority side is regarded as a binary fraction. Then, it is converted to a decimal fraction.

${ }^{2}$ These changes resemble the chaotic itinerancy proposed by Kaneko and Tsuda[10]. We still could not clarify whether the dynamics observed in our system is precisely chaotic itinerancy.
} 

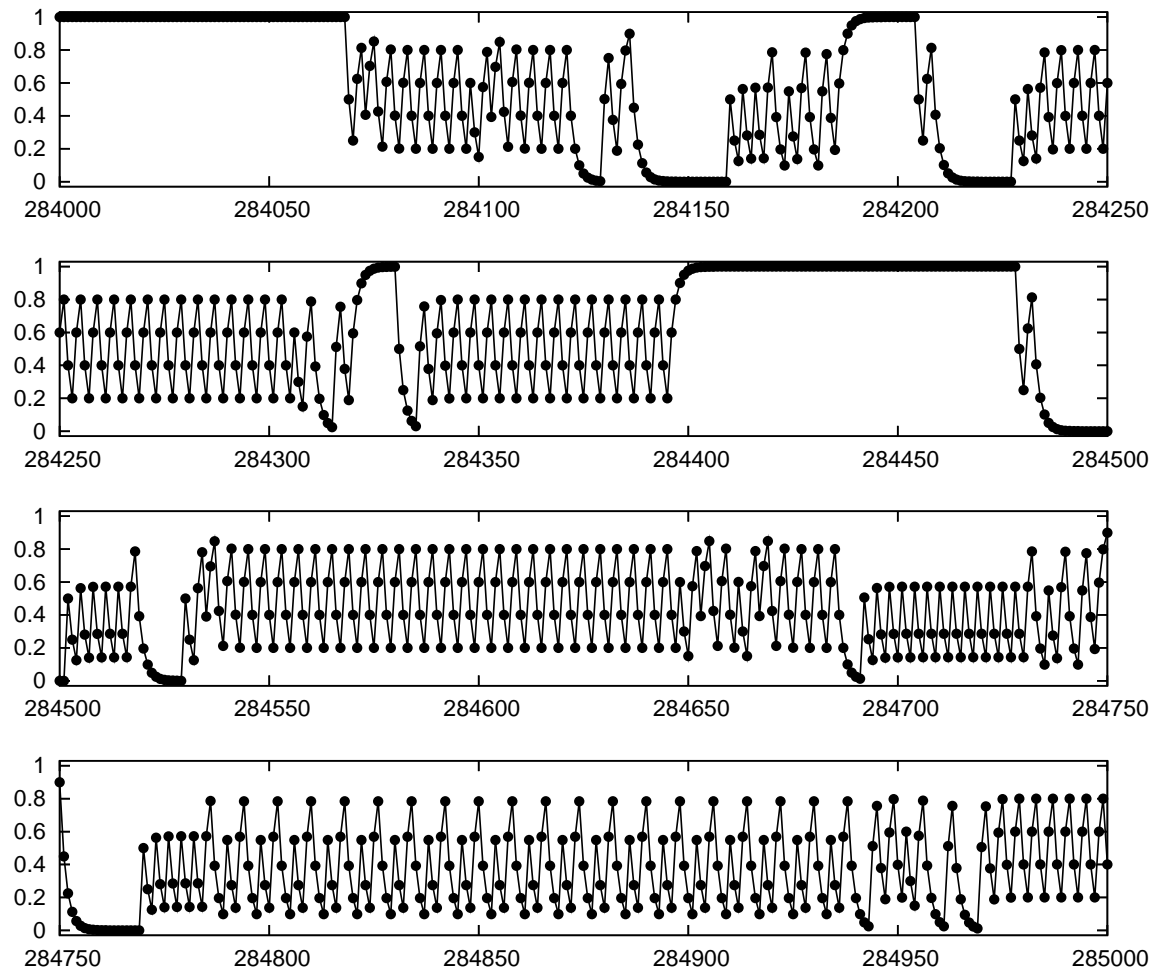

Figure 5: An example of itinerant dynamics at the macro level in one turn. The $x$-axis and the $y$-axis of each figure are the steps and the minority sides converted to real numbers, respectively. The dynamical states of the game change frequently among fixed points and various periodic cycles via aperiodic motions.

\subsubsection{Transition of Agent's Strategy}

We illustrate examples of the transitions of strategies in several turns in Fig. 7. These strategies vary through the learning process. An interesting agent behavior is found in the 24th turn. Although the minority side in this turn is fixed on ' -1 ,' that is, the same stimulus is continuously given to the agent, the trajectory of the agent's internal dynamics drawn in Fig. 7(a) shows a chaotic motion.

We conduct a correlation dimension analysis for the agent's output values and calculate lyapunov exponents for each embedding dimension in the analysis. The results of the analysis are shown in Fig. 8. By using the time series analyses, we confirm that the agent's motion is a low dimensional chaos with weak nonlinearity, since correlation dimension is about 0.92 , and lyapunov exponent is around 0.02 , when embedding dimension is 17 . In contrast, the other agent in the same turn depicted in Fig. 7(b) acquires a simple deterministic finite automaton with three states. In this turn, these agents alter their actions depending on only their past actions. In other words, they attain one-to-many relationships between an input and outputs. 

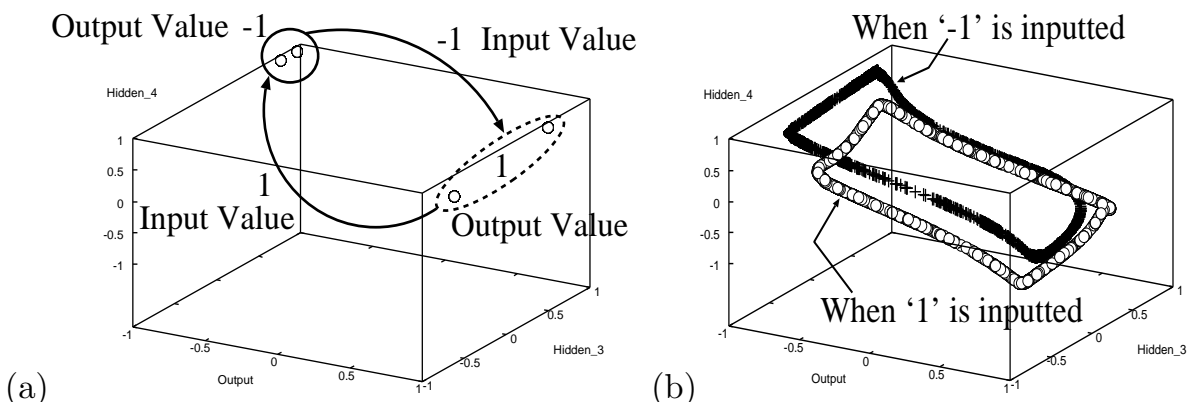

Figure 6: Strategies shown in a phase space. The $x, y$ and $z$-axes of each figure are the values of the output neuron, the third hidden neuron and the fourth hidden neuron, respectively. (a) A strategy described by a simple deterministic finite automaton with two states is depicted in the phase space. The small circles show the network states. The large circles and the arrows stand for the output values of the agent and the input value that he/she receives, respectively. The dotted circle is the initial state of the agent. The agent behaves periodically. (b) A strategy has two limit cycles corresponding to two input values. This means that the agent has a strategy that switches two output sequences according to the external stimuli.

\section{Discussion}

In this section, we discuss the implication and the causes of the itinerant dynamics. At first, we insist that the itinerant dynamics can be interpreted as the dynamics of macro structure in the agents' society. Then, we show that the micro-macro loop is crucial for forming and maintaining the dynamics at the macro level. Further, causes of the macro destabilization are argued. At last, we consider a difference between our proposed SRN-SIC and the original SRN.

\subsection{Itinerant Motion as Dynamics of Macro Structure}

Time series of the minority side show definite features such as fixed points and periodic motions. This suggests that the agents have certain internal structures and form certain relationships with other agents, because the time series of the minority side is decided by the sequence of all agents' moves. Besides, since fixed points and periodic cycles can be described by some rules of dynamical systems, agents' society showing such dynamics is considered as in some structuralized states with macro level rules. Accordingly, the feature of time series can reflect a macro structure in the agents' society.

In our system, the feature of the time series changes with time, as shown in Fig. 9. That is to say, the system realizes the dynamics of the macro structure in the agents' society, such as a labeled sequence of A, B and C representing social states in Fig. 9. Itinerant dynamics between fixed points and various periodic motions via aperiodic motions is not shown in other studies of the minority game. 
(a)
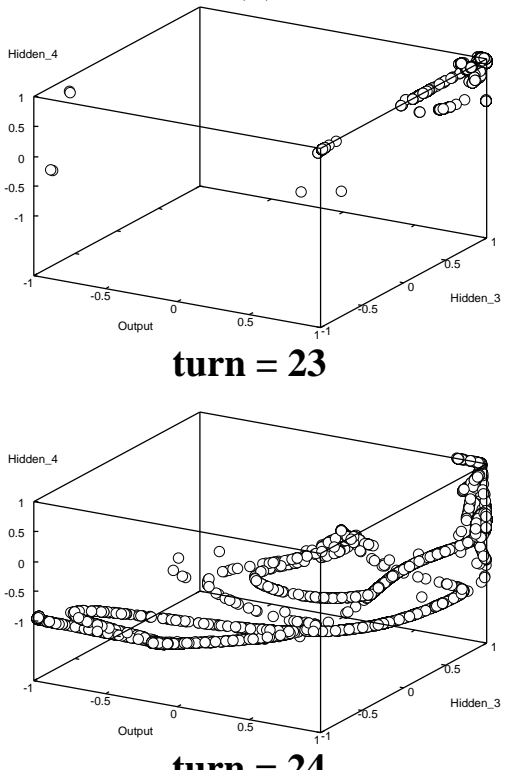

turn $=24$

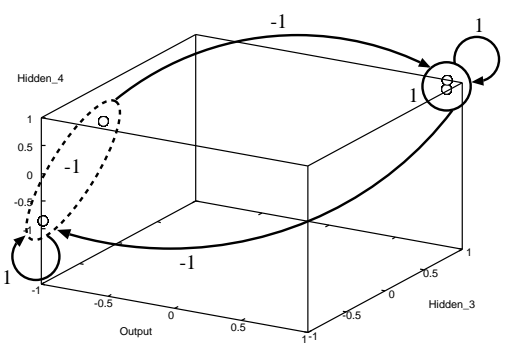

turn $=25$

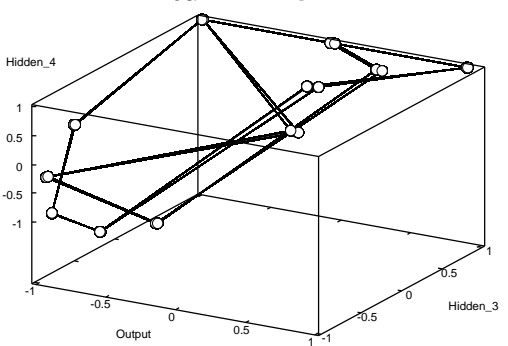

turn $=26$ (b)
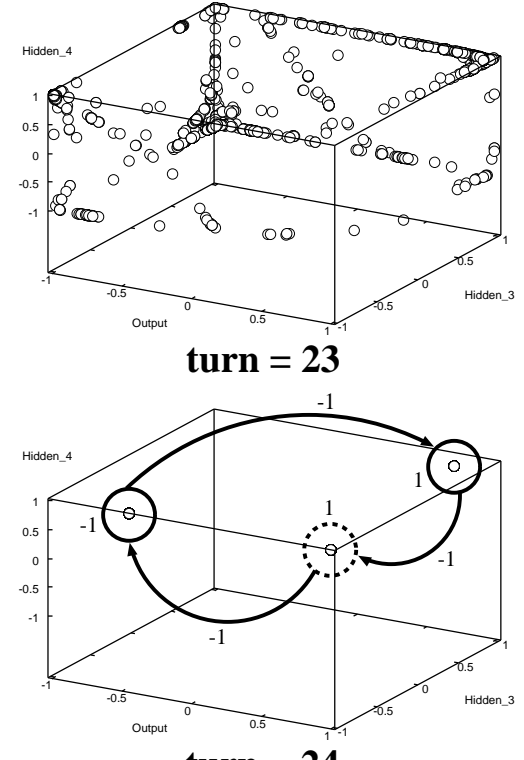

turn $=24$

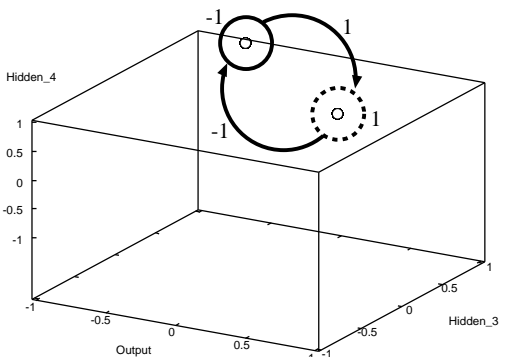

turn $=25$

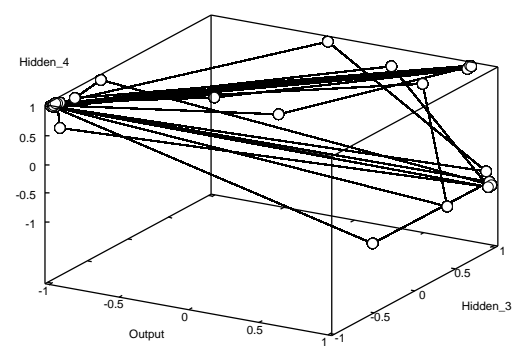

turn $=26$

Figure 7: Transition of two agents' strategies in turns 23 26. All axes are the same as those in Fig. 6 . The strategies of the agents vary through the learning process among various finite state automata and complex forms like strange attractors. Different agents have quantitatively different strategies in one turn. For example, in the 24th turn the strategy in (a) forms a strange attractor and in (b) a three states automaton. Although both of the strategies in (a) and (b) in turn 26 are finite automata with 12 and 30 states, respectively, the number of the states is too many to illustrate. Therefore, we draw the trajectories instead of circles and arrows. 


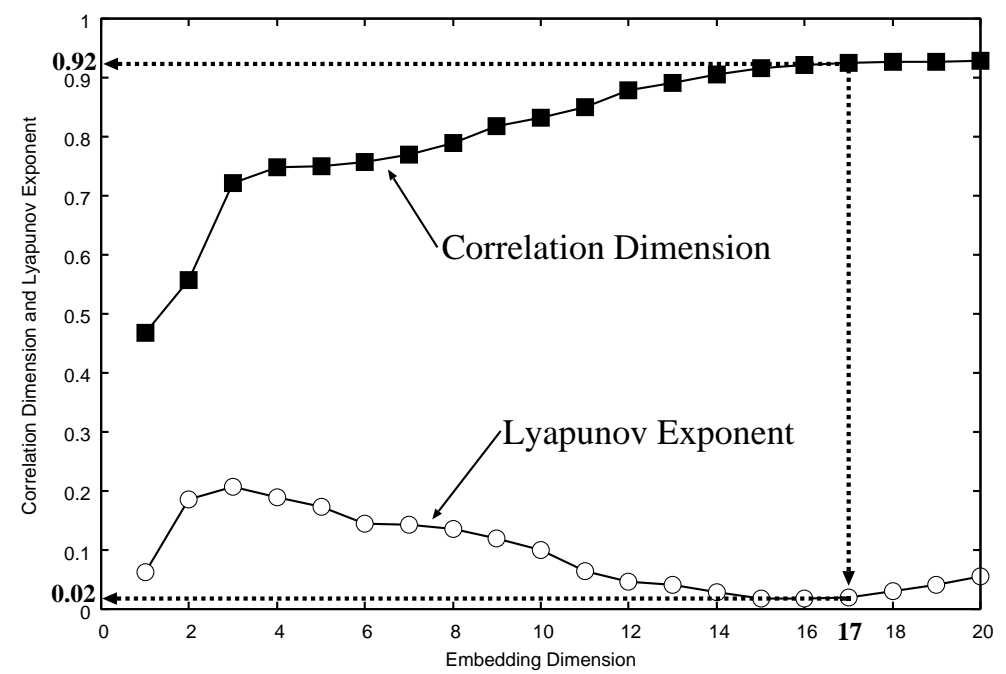

Figure 8: Correlation dimensions and lyapunov exponents against embedding dimension of the agent's output values in the 24th turn. It is clarified that this agent's dynamics is a low dimensional chaos with weak nonlinearity.

\subsection{Effect of Micro-Macro Loop}

The micro-macro loop is explicitly equipped with our system, since it is thought that the micro-macro loop is one of the source of macro dynamics. The effect of the micro-macro loop, however, is not confirmed yet. We conduct some experiments in order to argue the effect of the micro-macro loop. Remind that the micro-macro loop is implemented by the following manner: As the influence from the micro to the macro, the macro level is determined from all of the agents' move. As the influence from the macro to the micro, the minority side at the last step is given to all the agents as an external stimulus. In the following experiments, so as to cut this loop, the agents are forced to receive artifactual sequences as the external stimuli. Here, artifactual means that the agents receive the different input from the minority side at the last step. The experiments consist of: 1) Fixed or random sequences are input to the agents showing the itinerant dynamics at the macro level. 2) The itinerant sequence is input to the agents showing the fixed or periodic motions at the macro level.

\subsubsection{Fixed or Random Input to Agents with Itinerant Macro Dy- namics}

The itinerant dynamics is an interesting motion at the macro level. To clarify what is required in order to form and maintain the itinerant dynamics, we investigate how motions at the macro level change, when each agent continuously receives artifactual inputs instead of the past minority side as an external stimulus. Here, we give two types of artifactual input, 1) fixed sequence -1.0, 0.0, or $1.0,2)$ uniform random sequence with various ranges. Inputting such sequence to each agent means that a macro level has directly no influence on a micro level. We give the sequences to all agents in the middle of a turn that shows the 


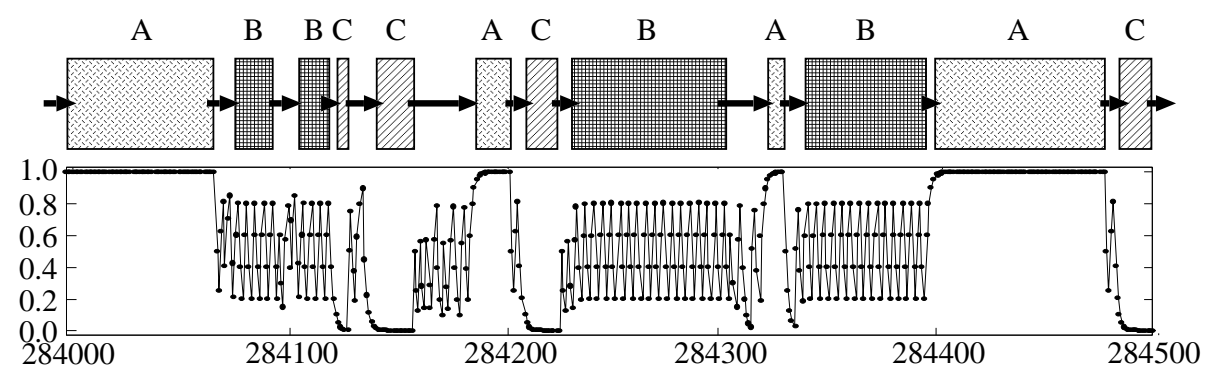

Figure 9: Structuralized states with macro level rules. The $x$-axis and the $y$-axis of figure are the steps and the minority sides converted to real numbers, respectively. Simple dynamics such as fixed point and periodic motion are labeled by $\mathrm{A} \sim \mathrm{C}$. The labels depict distinct macro structures in the agents' society. It is clear that spontaneous changes of the macro structures take place.

itinerant dynamics at macro level. The itinerant dynamics is shown from the 280,000 to the 290,000 steps, namely, the 28 th turn. To observe the dynamics for a long term, we extend the length of the turn until 1,000,000 steps. That is, we do not carry out learning process between the 280,000 and the 1,280,000 steps. In the extended turn, we give the information from the 780,000 steps to the 1,000,000 steps.

Figure $10(\mathrm{a}) \sim(\mathrm{c})$ show transitions of the minority side when all agents receive fixed numbers such as $-1.0,0.0$ or 1.0, respectively. The left-hand and the right-hand of Fig. 10(a) (c) are around the beginning (779,750 780,250 steps) and around the ending $(999,750 \sim 1,000,250$ steps) of the artifactual input, respectively. As can be seen, the itinerant dynamics collapses when starting to substitute the artifactual fixed input for the past minority side shaped by all agents. The itinerant dynamics, however, is regenerated by stopping the fixed input and affording the minority side at the immediately preceding play again, as illustrated in the right-hand of Fig. $10(\mathrm{a}) \sim(\mathrm{c})$. Therefore, the complete influential loop between the micro and the macro levels, namely, the micro-macro loop, plays an important role to produce and maintain the itinerant dynamics at the macro level.

In particular, it seems that all agents are strongly controlled by -1.0 and 1.0 as fixed external stimulus, since the motions at macro level are attracted toward these values, as illustrated in Fig. 10(a) and (c). This means that all agents can be regulated by external stimulus to some extent. In other words, we may be able to dominate dynamics of macro structures by controlling input information to all agents.

In case that uniform random numbers are given to all agents, collapse of the itinerant dynamics occurs by inputting the random number to the agents as illustrated in the left-hand of Fig. 11(a) (c), and regeneration of the itinerant dynamics endogenously takes place by restoring the micro-macro loop without exception as illustrated in the right-hand of Fig. 11(a) (c), as well as the previous experiments giving the artifactual fixed inputs to the agents.

Through the experiments that all agents receive the various external stimulus instead of the past minority side shaped by themselves, we confirm that the micro-macro loop is necessary to produce and maintain the itinerant dynamics 
(a) -1.0 is given.
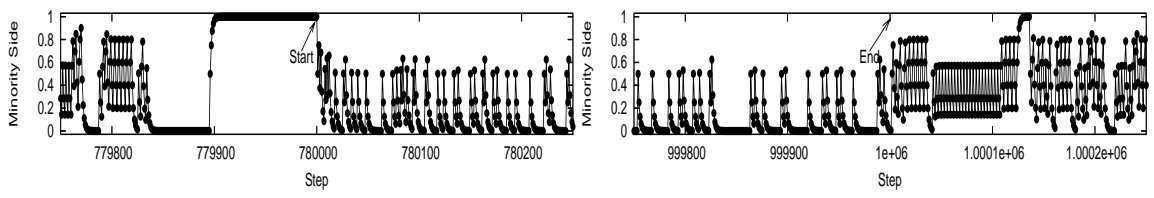

(b) 0.0 is given.
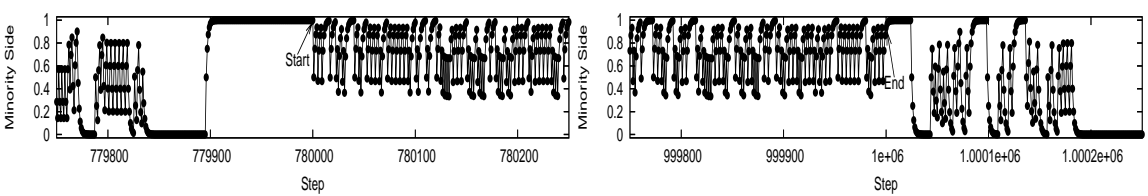

(c) 1.0 is given.
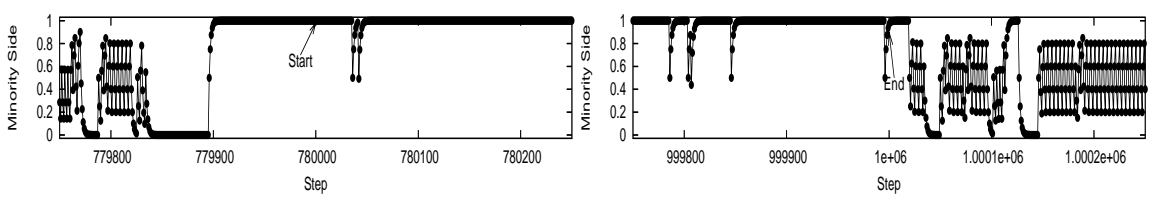

Figure 10: Collapse and regeneration of the itinerant dynamics with starting and ending of the artifactual fixed inputs. The $x$-axis and the $y$-axis of each figure are the steps and the minority sides converted to real numbers, respectively. These figures are transitions of the minority side in case that each agent receives (a)-1.0, (b)0.0 and (c)1.0 as external stimulus at the every step. The left-hand figures are in the 779,750 780,250 steps, around the beginning of the fixed inputs. The right-hand figures are in the 999,750 1,000,250 steps, around the ending of the fixed inputs. Each fixed input is given to all agents between the 780,000 , indicated by a labeled arrow 'Start', and the 1,000,000 steps, indicated by a labeled arrow 'End'. The itinerant dynamics are eliminated by inputting such sequences. But, the dynamics are regenerated by all agents again, when they start to receive the past minority side shaped by them.

at the macro level.

\subsubsection{Itinerant Input to Agents with Fixed or Periodic Macro Dy- namics}

In order to investigate the effect of itinerant dynamics itself, we observe how motions at the macro level change in case that the agents receive the time series of itinerant dynamics. In these experiments, we give the itinerant macro dynamics shaped by the agents at the 28th turn (shown in Fig 5) to all agents as external stimuli from the beginning to the half of one turn, in which the macro dynamics with normal external stimuli is fixed point or periodic motion. Consequently, while inputting the time series of the itinerant dynamics, different itinerant dynamics from the input sequence is often shaped. An example of formation of the different itinerant dynamics is illustrated in Fig. 12(a). The itinerant dynamics is not always formed. There are some cases that fixed point with occasional fluctuation emerges, as shown in Fig. 12(b). Further, there are some instances that the original fixed points at the macro level are not quite 
(a) Random number between -0.001 and 0.001 is given.
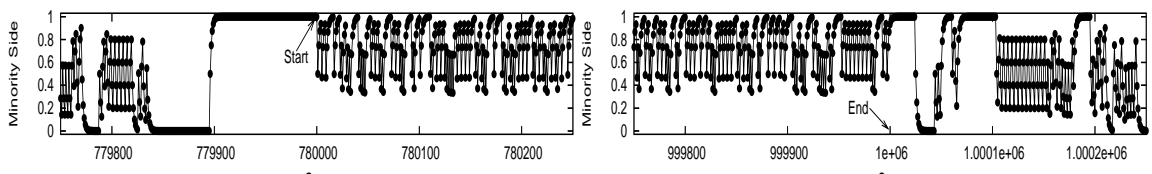

(b) Random number between -0.5 and 0.5 is given.
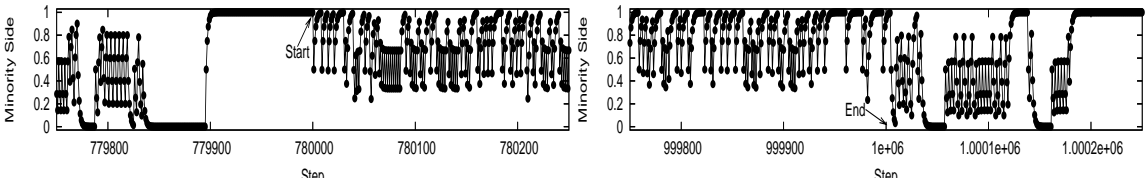

(c) Random number between -1.0 and 1.0 is given.
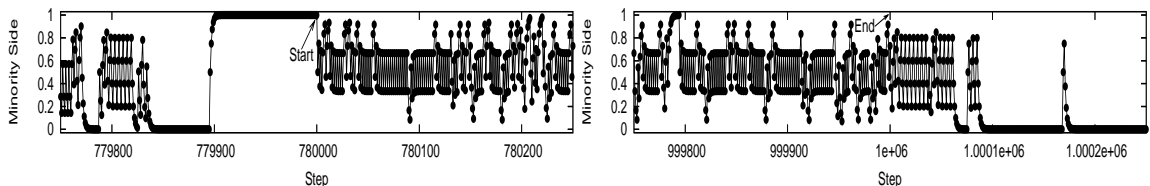

Figure 11: Collapse and regeneration of the itinerant dynamics with starting and ending of the artifactual uniform random inputs having various range. The $x$-axis and the $y$-axis of each figure are the steps and the minority sides converted to real numbers, respectively. These figures are three examples of transitions of the minority side in case that each agent receives the random number (a) between -0.001 and 0.001 , (b) between -0.5 and 0.5 and (c) between -1.0 and 1.0 as external stimulus. The condition for the random inputs and the labels are the same as Fig. 10. The itinerant dynamics are eliminated by inputting the random sequences and regenerated by stopping the random inputs.

changed. Thus, there is no assurance that the itinerant dynamics is surely shaped at the macro level even if the time series of the itinerant dynamics is given to all agents.

Moreover, in almost all cases, when each agent stops to receive the time series of itinerant dynamics, namely, begins to receive the past minority side shaped by themselves again, the original dynamics shown before receiving the itinerant dynamics as external stimulus is regenerated ${ }^{3}$, as illustrated in Fig. 12. That is, the itinerant dynamics cannot be maintained unless the itinerant dynamics is continuously given to each agent, even if the itinerant dynamics was shaped from the inputted itinerant dynamics. Therefore, it is thought that the micro-macro loop has functions to not only produce the itinerant dynamics but also maintain it. This results suggest that agents with specific structure are necessary in a micro level so that dynamics of macro structure is endogenously shaped at a macro level.

\footnotetext{
${ }^{3}$ Different dynamics from the original dynamics are sometimes shaped by the agents as exceptional cases.
} 
(a)

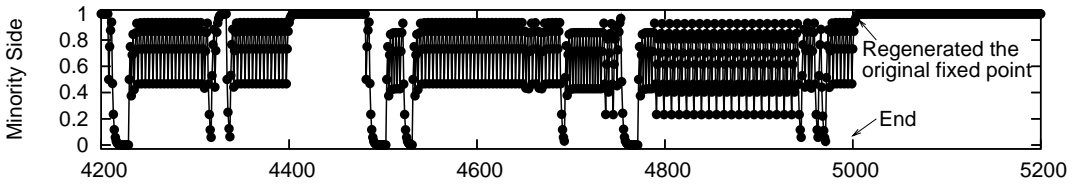

a)

Step

(b)

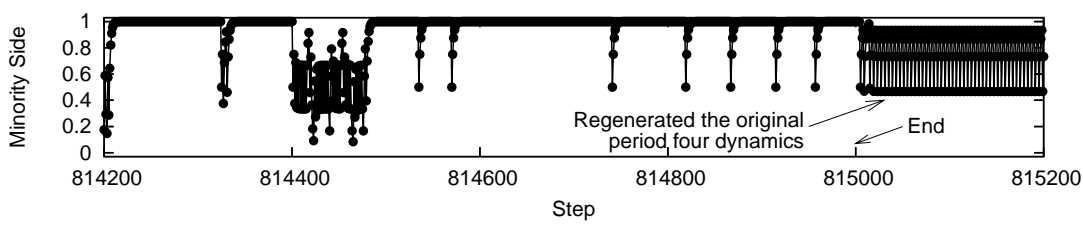

Figure 12: Time series of the minority side, while inputting the itinerant dynamics to the agents with fixed or periodic macro dynamics. The $x$-axis and the $y$-axis of each figure are the steps and the minority sides converted to real numbers, respectively. Figure (a) is an example of itinerant dynamics. This itinerant dynamics is different from the input sequence. Figure (b) is an example of a fixed sequence with occasional fluctuation. In both cases, the original dynamics, (a) fixed point and (b) period four, are regenerated by stopping the itinerant inputs.

\subsection{Causes of Dynamics at Macro Level}

It is thought that the dynamics of the macro structure is brought about by some instability in the system. If so, where is the instability? From the result showing different features of the time series for each turn (Fig. 4), instability must be caused by the learning between turns. Further, there seems to be another instability that is produced by the internal dynamics and interaction of the agents, since the system itinerate among various dynamical states in one turn (Fig. 5). In the following sections, we discuss both of the instabilities.

\subsubsection{Learning under Static Assumption}

In the learning process, each agent independently forms a prediction model from the sequence of minority side of the past 100 steps, to estimate the transition of the game in the next turn. All agents try to predict the macro structure in the future from a part of the past events. This prediction implies that the agents assume the basically unchanged or static structure. The structure, however, is constructed by all agents whose behavior is modified by the learning process. Therefore, a prediction model generated under the static assumption that the past structure is preserved as is does not work well to predict the transition of the game.

This is structurally the same destabilization mechanism that is seen in Taiji and Ikegami's studies of the coupled dynamical recognizers[18, 9]. There are two agents playing the iterated prisoners' dilemma game in their model. The agents try to make models of their opponents mutually through learning. For each agent, the opponent's model used in the previous game is often different from the current opponent. Thus, the dynamics of the game becomes unstable, since the model cannot predict the current opponent's move correctly. If the 
both agents, however, predict their opponent as a simple finite state automaton, the dynamics of the game can be stabilized for a certain period. When either of the agents becomes unstable, the other agent is destabilized by reacting to opponent's change sensitively. Our model can be thought of as an extension from the relationship between two persons in the model of Taiji and Ikegami[18, 9] to that among many people. Even though only the moves of the minority side are input to each agent, the minority side is constructed from the moves of all agents. Accordingly, each agent relates to all agents indirectly.

The prediction under the static assumption is an expression of the agent's bounded rationality. In actual societies, no one can make a complete prediction model that takes the consequences of behavioral changes of all people into consideration. Therefore, the cause of destabilization discussed here is inevitable when social structures are endogenously formed.

\subsubsection{Chaotic Actions implying One-to-Infinity Relationship}

To know what feature at the micro level causes the itinerant dynamics at the macro level in one turn, we investigate configuration of the agents' actions at the micro level. Table 2 shows the configuration corresponding to the classification of the dynamics introduced in section 4.1. When the itinerant dynamics is shown at the macro level, the number of agents with aperiodic actions is much larger than the other cases. The aperiodic action may be chaotic dynamics as indicated in section 4.2. Chaotic dynamics has orbital instability, which expands small differences in the trajectories of agents' actions. Therefore, even a small displacement at the micro level can induce a change in the macro level dynamics.

Let us discuss the aperiodic action from the viewpoint of the relationship between inputs and outputs. A strategy with one-to-many relationships can emit aperiodic actions. Periodic actions, however, are also derived from a strategy with one-to-many relationships, as shown in Fig.7(b), the 24th turn. The distinction of these strategies is that the one with aperiodic action, characterized by strange attractors, forms potentially a one-to-infinity relationship from an input to outputs. Accordingly, the condition for the dynamics of macro structure to appear may be that there exists a certain number of agents having a one-to-infinity relationship between an external stimulus and their actions. Internal dynamics is indispensable for obtaining such complex behavior.

\subsubsection{Threshold in Minority Game}

A feature of the minority game also can be thought of as the cause of destabilization. In the minority game, there is a threshold at the half of the player population. The result of the game changes if only a few players alter their behavior, when the number of agents in the minority side is around the threshold. The similar threshold effect works when the output value of the SRN-SIC is divided into '-1' or ' 1 .' Thus, behavior of the SRN-SIC easily changes by a small fluctuation when the output value is around 0.0. 
Table 2: The correspondence between dynamics at the macro level and the configuration of agents at the micro level. The left-hand side of the table indicates the classification at the macro level dynamics (see section 4.1). The first and the second columns are the dynamical states of the minority side and that of the number of winners, respectively. Each number in the right-hand side of the table stands for the number of agents whose actions are in the specific dynamical states specified at the second row. In the case of itinerant macro dynamics (the bottom row), the number of agents showing aperiodic actions is much larger than in the other cases.

\begin{tabular}{|c|c||c|c|c|}
\hline \multicolumn{2}{|c||}{ Macro Level } & \multicolumn{3}{c|}{ Micro Level } \\
\hline $\begin{array}{c}\text { Minority } \\
\text { Side }\end{array}$ & $\begin{array}{c}\text { The number } \\
\text { of Winners }\end{array}$ & $\begin{array}{c}\text { Fixed } \\
\text { Point }\end{array}$ & $\begin{array}{c}\text { Periodic } \\
\text { Motion }\end{array}$ & $\begin{array}{c}\text { Aperiodic } \\
\text { Motion }\end{array}$ \\
\hline \hline $\begin{array}{c}\text { Fixed } \\
\text { Point }\end{array}$ & $\begin{array}{c}\text { Fixed } \\
\text { Point }\end{array}$ & 101 & 0 & 0 \\
\hline $\begin{array}{c}\text { Fixed } \\
\text { Point }\end{array}$ & $\begin{array}{c}\text { Periodic } \\
\text { Motion }\end{array}$ & 84 & 17 & 0 \\
\hline $\begin{array}{c}\text { Fixed } \\
\text { Point }\end{array}$ & $\begin{array}{c}\text { Aperiodic } \\
\text { Motion }\end{array}$ & 50 & 46 & 5 \\
\hline $\begin{array}{c}\text { Periodic } \\
\text { Motion }\end{array}$ & $\begin{array}{c}\text { Periodic } \\
\text { Motion }\end{array}$ & 63 & 38 & 0 \\
\hline $\begin{array}{c}\text { Periodic } \\
\text { Motion }\end{array}$ & $\begin{array}{c}\text { Aperiodic } \\
\text { Motion }\end{array}$ & 20 & 77 & 4 \\
\hline $\begin{array}{c}\text { Itinerant } \\
\text { Motion }\end{array}$ & $\begin{array}{c}\text { Itinerant } \\
\text { Motion }\end{array}$ & 8 & 6 & 87 \\
\hline
\end{tabular}

\subsection{Difference between SRN-SIC and SRN}

In our proposed SRN-SIC, a self-influential connection is added to the SRN, because humans can often recognize their own actions performed intentionally and can make self-examinations. Thus, there is clearly a conceptual difference between the SRN-SIC and the SRN. There also exists a mathematical difference between them. The SRN-SIC has higher-order nonlinearity, since the past information passed to the hidden layer is transformed twice by a nonlinear function, while the information stored internally by the context layer is transformed once in one step.

To know phenomenological difference between the SRN-SIC and the SRN, we compare the macro dynamics shown in the society of each type of agents. As a consequence, the number of times with aperiodic macro motion is 1137 times per 10000 turns in the experiments using the SRN-SIC, as illustrated in Fig. 13(a) against 654 times in the experiments using the SRN, as illustrated in Fig. 13(b). That is to say, it is confirmed that the SRN-SIC has a certain capability to make a macro level complex than the SRN.

Note that the itinerant dynamics does not take place in all experiments using 

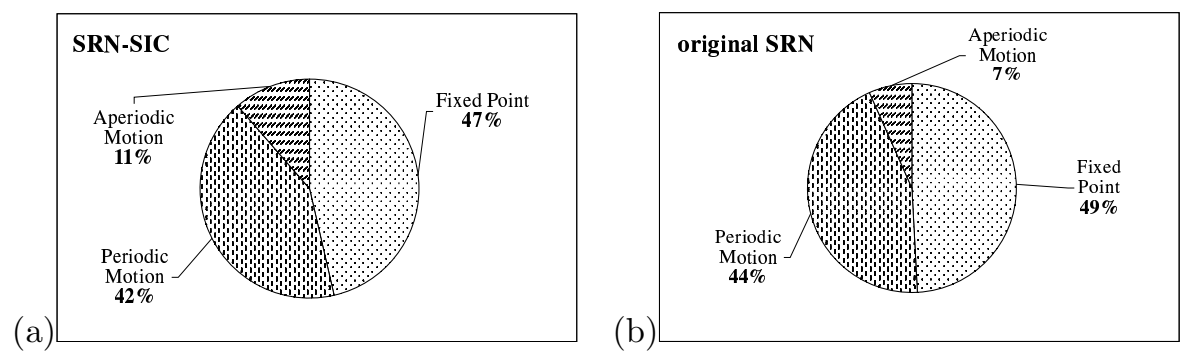

Figure 13: Frequency of occurrence of three patterns such as fixed point, periodic motion and aperiodic motion at the macro level. (a) and (b) are the results of SRN-SIC and that of SRN, respectively. In particular, it is clarified that the incidence of aperiodic motion at macro level is $11 \%$ in the experiments using (a)the SRN-SIC against 7\% in the experiments using (b)the SRN.

the SRN. Therefore, being influenced by the past own behavior, namely, the selfinfluential connection, may have a certain effect in formation of the itinerant dynamics at the macro level.

\section{Conclusion}

In this paper, we have discussed the effectiveness of viewing a human as a cognitive agent having internal dynamics when we consider the emergence and dynamical changes of social structures. We proposed a model of a social agent having internal dynamics in terms of an simple recurrent network with selfinfluential connection (SRN-SIC) in order to illustrate the importance concretely. By using a dynamic social simulation equipped with a micro-macro loop and composed of the agents which we have proposed, we have shown that complex dynamics at both a micro and a macro level took place.

We have obtained results that showed the dynamics of macro structures. In particular, it is interesting that, though the agents' internal structures are not modified by learning, the macro structure shows an itinerant dynamics, which is an aperiodic change among ordered states such as fixed point and periodic motion through disordered states. It is also interesting that the agents exhibit complex behavior despite of fixed external environment.

These simulation results substantiate the significance of internal dynamics for forming and maintaining a dynamic social structure. Further, it is shown that the micro-macro loop is necessary to produce and maintain the dynamics of macro structures. Thus, we conclude that internal dynamics and micromacro loop are necessary to form and maintain a dynamic social structure. To construct dynamic social simulations, we also argue that our proposed SRNSIC is an efficient architecture of a social agent having internal dynamics. It is especially significant to adopt such agent having the internal dynamics in order to study dynamic nature of society with multi-agent systems, instead of static agents often employed.

The sources of the dynamics of macro structures are conjectured as follows. Firstly, each member does not consider the behavioral change of all members 
to predict the future constructed by all members; secondly, there is a oneto-infinity relationship between an external stimulus and the actions of each member. We confirmed the SRN-SIC is more effective to produce complex macro dynamics than the original SRN. However, it is not clarified throughly how the itinerant dynamics emerged, and how the difference of the models is brought, in detail. In further studies, we will clarify the influence of the internal dynamics on agents' behavior and on the macro structure. Furthermore, we will analyze an effect of the self-influential connection in the SRN-SIC in order to demonstrate effectiveness of the SRN-SIC as dynamic social agent. By solving these problems, we will be able to well perform dynamic social simulations which can analyze the essence of the dynamics in actual societies.

\section{Acknowledgement}

The authors wish to thank Susumu Egashira, Takashi Iba, Jun Namikawa and Gen Masumoto for their fruitful discussions. This research was partially supported by a Grant-in-Aid for Scientific Research (No.15700183) from the Ministry of Education, Culture, Sports, Science and Technology of Japan and by the Japan Society for the Promotion of Science.

\section{References}

[1] Aoki, M. (2001). Towards a Comparative Institutional Analysis. Cambridge, MA: MIT Press.

[2] Challet, D., \& Zhang, Y. C. (1997). Emergence of cooperation and organization in an evolutionary game. Physica A, 246, 407-418.

[3] Egashira, S., \& Hashimoto, T. (2004). A position of human cognition in social science (in Japanese). In M. Nishibe (Ed.), A Frontier of Evolutionary Economics (pp. 159-180). Tokyo: Nihon-Hyouron-Sha.

[4] Elman, J. L. (1990). Finding structure in time. Cognitive Science, 14, 2, $179-211$.

[5] Elman, J. L. (1991). Distributed representations, simple recurrent networks, and grammatical structure. Machine Learning, 7, 195-225.

[6] Elman, J. L. (1993). Learning and development in neural networks: the importance of starting small. Cognition, 48, 71-99.

[7] van Gelder. T. (1998). The dynamical hypothesis in cognitive science. Brain and Behavioral Sciences, 21, 615-665.

[8] Hashimoto, T., \& Egashira, S. (2001). Formation of social norms in communicating agents with cognitive frameworks. Journal of Systems Science and Complexity, 14, 1, 54-74,

[9] Ikegami, T., \& Taiji, M. (1999). Imitation and cooperation in coupled dynamical recognizers. In D. Floreano, et al (Eds.), Advanced in Artificial Life (pp. 545-554). Berlin: Springer-Verlag. 
[10] Kaneko, K., \& Tsuda, I. (2003). Chaotic itinerancy, chaos: focus issue on chaotic itinerancy. Chaos, 13, 3, 926-936.

[11] Karmiloff-Smith, A. (1992). Beyond Modularity: A Developmental Perspective on Cognitive Science. Cambridge, MA: MIT Press.

[12] Nagao, N., Nishimura, H., \& Matsui, N. (2000). A neural chaos model of multistable perception. Neural Processing Letters, 12, 3, 267-276.

[13] Nishimura, H., Nagao, N., \& Matsui, N. (1997). A perception model of ambiguous figures based on the neural chaos. In Kasabov, et al (Eds.), Progress in Connectionist-Based Information Systems (pp. 89-92), 1, Berlin: Springer-Verlag.

[14] Pollack, J.B. (1991). The induction of dynamical recognizers. Machine Learning, 7, 227-252.

[15] Port, R., \& van Gelder. T. (1995). Mind as Motion: Explorations in the Dynamics of Cognition. Cambridge, MA: MIT Press.

[16] Shiozawa, Y. (1997). Consequences of Complexity (in Japanese). Tokyo: NTT Shuppan.

[17] Shiozawa, Y. (1997). An Introduction to the Economics of Complexity (in Japanese). Tokyo: Seisansei Shuppan.

[18] Taiji, M., \& Ikegami, T. (1999). Dynamics of internal models in game players. Physica D, 134, 253-266.

[19] Tani, J., \& Nolfi, S. (1998). Learning to perceive the world as articulated: an approach for hierarchical learning in sensory-motor systems. Neural Networks, 12, 1131-1141.

[20] Varela, F.J., Thompson, E., \& Rosch, E. (1991). The Embodied Mind Cognitive Science and Human Experience-. Cambridge, MA: MIT Press.

[21] Zhang, Y.C. (1998). Modeling market mechanism with evolutionary games. Europhys. News, 29, 51-54. 